\title{
Research of Vortex Nucleation in Bose-Einstein Condensates
}

\author{
Li Ji, Ren Yuan*, Shao Qiongling \\ Department of Spaceflight Equipment, The Academy of Equipment, Beijing, China
}

Email address:

mingji_xinlang@sina.com (Li Ji), renyuan_823@aliyun.com (Ren Yuan), zzy5678@126.com (Shao Qiongling)

${ }^{*}$ Corresponding author

\section{To cite this article:}

Li Ji, Ren Yuan, Shao Qiongling. Research of Vortex Nucleation in Bose-Einstein Condensates. Science Discovery.

Vol. 5, No. 1, 2017, pp. 1-6. doi: 10.11648/j.sd.20170501.11

Received: December 10, 2016; Accepted: January 10, 2017; Published: March 15, 2017

\begin{abstract}
Vortex nucleation is the key technology to realize quantum gyroscope in Bose-Einstein Condensates (BEC). Through mass investigation and research, the recent research status of vortex nucleation in Bose-Einstein Condensates is presented, the theory and experimentation about imprinting phase, rotating the potential trap and transferring the orbital angular momentum of a photon to generate BEC vortex are analyzed and compared in detail, furthermore, an improved method of rotating the potential trap to generate vortex is proposed. Quantum gyroscope based on BEC vortex has the potential advantages of realizing the ultra high sensitivity and small volume of quantum gyroscope. This paper aims to promote the development of quantum vortex gyroscope, which attracts extensive attention of domestic scholars.
\end{abstract}

Keywords: BEC, Vortex Nucleation, Rotating the Potential Trap, Quantum Vortex Gyroscope

\section{BEC浴旋成核方法研究}

李基, 任元 ${ }^{*}$, 邵琼玲

装备学院航天装备系, 北京, 中国

邮箱

mingji_xinlang@sina.com（李基），renyuan_823@aliyun.com（任元），zzy5678@126.com（邵琼玲）

摘要: 玻色-爱因斯坦凝聚（Bose-Einstein Condensation，简称BEC）浴旋成核是实现BEC浴旋陀螺效应的关键技术。 通过对BEC浴旋成核相关理论和实验方法的调研和分析, 本文阐述了BEC浴旋特性的研究现状, 对BEC浴旋成核的印刻相 位法、旋转势阱法和传递角动量法的工作原理和实验方法进行详细研究和对比分析, 提出了一种改进型旋转势阱法的 涡旋成核方法, 并指出BEC浴旋的陀螺效应具有实现超高灵敏度、小体积量子陀螺仪的潜在优势。本文旨在促进量子浴 旋陀螺仪的发展, 引起国内学者的广泛关注。

关键词：BEC，浴旋成核，旋转势阱，量子浴旋陀螺

\section{1. 引言}

惯性导航系统是一种无需外接交互的自主导航系统, 具有抗干扰能力强、隐蔽性强以及全天时全天候工作的特 点, 作为导航领域的研究热点始终备受关注。陀螺仪是惯 性导航系统的重要核心，现有陀螺技术在超高灵敏度精度、
小体积、低功耗之间存在矛盾，限制了现有惯性测量技术 的进一步发展, 因此, 亟需探索新的陀螺技术。目前新技 术陀螺仪的研究方向主要是基于低温物理效应的量子干 涉陀螺。量子干涉陀螺的工作原理是物质波的Sagnac效应, 与光子的Sagnac干涉效应类似, 基于Sagnac效应的量子干 涉陀螺灵敏度同样存在局限性, 它受到干涉面积的制约, 
因而其高灵敏度精度与小体积之间存在矛盾, 这一矛盾在 原理上无法避免。在精度需求特别高的战略级应用场合, 机械转子式陀螺仪仍然是首选对象, 但是由于摩擦和漂移 等误差的存在, 仍然无法达到超高灵敏度精度、小体积、 低功耗的最优配置。

爱因斯坦最早预言，在温度足够低的条件下，玻色粒 子将发生相变, 即全部玻色子会凝聚到相同的最低能级上, 这就是最早的玻色爱因斯坦凝聚（BEC）表述 [1]。BEC是 超流体的一种, 超流体是一种特殊的物质状态, 它具有内 部无粘滞、无摩擦碰撞等特点; 量子浴旋是超流体的重要 特性, BEC量子浴旋可以通过增加原子数而携带较大的轨 道角动量, 这些条件都比较符合新型转子的特点。实验发 现 [2], BEC涡旋具有进动特性, 这为新型陀螺仪的探索和 研究带来了巨大希望。

BEC 的浴旋成核是实现BEC浴旋陀螺效应的前提和基 础, 本文详细论述和分析目前国际上BEC浴旋成核的相关 理论和具体实验方法, 旨在填补我国量子㵀旋领域研究的 空白, 同时推动国内外专家学者交流, 促进量子浴旋陀螺 仪技术的探索和发展。

\section{BEC浴旋的研究现状}

浴旋是一种带有奇点的拓扑激发 [3], 稳定的浴旋态 是超流体的重要标志。量子浴旋最早用于研究液氦的超流 性, 但是液氦中发生玻色凝聚的成分相当少, 大约占总数 的 $10 \%$ 左右, 且由于具有强相互作用和高密度的特点, 使 得理论解释变得比较复杂, 不利于实验和理论验证。在低 温下, 气态凝聚体的密度比较低, 原子间的相互作用比较 小, 为了解决此类系统的描述和解算问题, Gross 和 Pitaevskii提出了弱相互作用下的气体玻色子平均场理 论。气态BEC发生凝聚的成分可高达 $99 \%$, 且冷原子气体稀 薄纯净、原子自由度易于操控, 因此气态BEC更有利于研 究量子浴旋 [4]。对于玻色-爱因斯坦凝聚体而言, 当旋转 速度接近临界值时, BEC中便会出现大量涡旋, 来响应外 界的扰动, 因为体系的能量有限, 在浴旋核的对称中心处 原子密度为零。

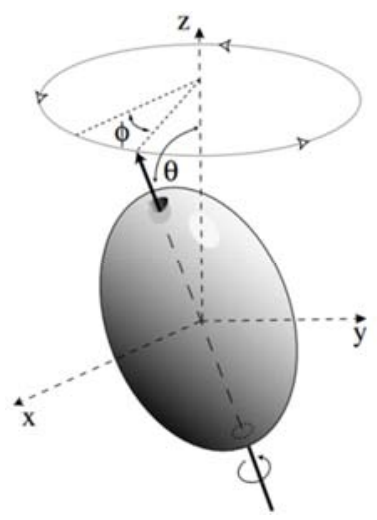

图1 BEC浴旋陀螺效应原理图 [5]。

意大利Trento大学的研究小组对BEC浴旋特性进行了 原理上的推导和理论分析 [5]。如图1所示, $X 、 Y 、 Z$ 轴 为势阱所在坐标系, 灰色椭球体表示处于势阱中的BEC浴
旋核, BEC 浴旋由于具有轨道角动量, 会保持其浴旋轴的 定向性。当势阱突然旋转使得浴旋轴倾斜 $\theta$ 角时, BEC浴 旋核便会发生进动现象, 进动角为图 1 中 $\phi$ 角。

随后, 英国牛津大学的E. Hodby 、S. A. Hopkins、 N. L. Smith等人从实验上证实了BEC浴旋的这一进动特性 [6], 得到的实验结果与理论预测基本相符。但实验中观 测到剪刀振荡现象, 并将剪刀振荡 [7]频率引入了浴旋进 动频率的表达式中, 使其更加完善, 最终得出浴旋核的进 动频率表达式为:

$$
\begin{aligned}
\Omega & =\frac{\left\langle L_{z}\right\rangle}{2 m\left\langle x^{2}+z^{2}\right\rangle} \\
& =\frac{7 \omega_{s c}}{2} \frac{\left\langle L_{z}\right\rangle}{N \hbar} \frac{\lambda^{5 / 3}}{\left(1+\lambda^{2}\right)^{3 / 2}}\left(15 N \frac{a}{a_{h 0}}\right)^{-2 / 5}
\end{aligned}
$$

式 (1) 中 $\left\langle L_{z}\right\rangle$ 是涡旋的角动量, $m$ 是原子的质量, $\left\langle x^{2}+z^{2}\right\rangle$ 是 $x$ 和 $z$ 的坐标值平方和的平均值, $N$ 是处于凝 聚态的总原子个数, $\omega_{s c}$ 是剪刀振荡频率, $\left\langle L_{z}\right\rangle=N \hbar\left(1-d^{2} / R_{r}\right)^{5 / 2}, \lambda=\omega_{z} / \omega_{r}, a_{h 0}=\sqrt{\hbar / m\left(\omega_{x} \omega_{y} \omega_{z}\right)^{1 / 3}}$ 代 表势阱谐波振荡长度, $a$ 表示s波散射长度, $\omega_{x}$ 和 $\omega_{y}$ 表示 $x$ 和 $y$ 方向上的势阱频率， $\hbar$ 是单粒子的角动量， $d$ 是浴 旋的径向位置, $R_{r}$ 是径向凝聚尺寸。

理论上, 基于 $\mathrm{BEC}$ 量子浴旋进动效应的量子浴旋陀螺 具备超高灵敏度精度陀螺仪的潜在优势, 同时为降低散粒 噪声的影响, 可以采用先进探测技术提高其灵敏度精度。 目前有几种待研究的检测方法, 如通过运用短波光或者近 场成像技术提高对BEC密度检测的敏感性; 利用高密度的 BEC云团提高散射光子数等方法可以提高灵敏度以及太赫 兹实时呈像技术等。

对BEC浴旋的特性研究还基于很多方面，2015年，美 国麻省理工学院的Wolfgang Ketterle经过理论分析和实 验验证, 从波函数动力学角度揭示了冷原子量子霍尔效应 的机理 [8]。同年, 上海纽约大学的Tim Byrnes和Jonathan P. Dowling等人对BEC中浴旋的量子霍尔效应进行了研究, 并提出一种使用拓扑不变量测量BEC中原子质量的新方法, 研究中也对量子浴旋电荷特性进行了大量分析 [9]。装备 学院于 2015年在国内首次提出量子浴旋陀螺的概念, 并与 北京航空航天大学、中科院物理所开展合作研究, 其研究 方向包括BEC浴旋的进动和干涉特性, 目前已具有制备BEC 的实验条件, 正在进行涡旋光实验平台的搭建, 建成以后 可成为国内首个利用浴旋光制备 $\mathrm{BEC}$ 量子浴旋的实验平台, 可用于对BEC浴旋成核的进动特性的研究, 以及 $\mathrm{BEC}$ 量子浴 旋陀螺的探索。

\section{BEC浴旋成核方法}

\section{1. 印刻相位法}

\section{(1) 工作原理}

E. A. Corne11和C. E. Williams 小组 [10]根据Williams 和Holland的理论 [11], 第一次在试验中从BEC气体原子中 
观察到浴旋现象。Williams和Holland的理论涉及到铷原 子的两种内部状态, 称为两种基态 ${ }^{87} R b$ 原子的超精细塞曼 亚能级态, 他们之间可以通过微波场互相转变 (分别用 $|1\rangle$ 和 $|2\rangle$ 表示），|1 状态和 $|2\rangle$ 状态的铭原子的散射长度不 相同。E. A. Corne11和C. E. Williams等人采用的实验方案 是基于对物质波函数的相干控制, 采用激光和射频场技术, 利用双组分 ${ }^{87} R b$ 的 $\mathrm{BEC}$ 气体原子产生浴旋。

(2) 实验方法与结果分析

E. A. Corne11和C. E. Williams 小组实验原理图如图2 所示。首先产生处于 $|1\rangle$ 状态下的 ${ }^{87} R b$ 原子气体 $\mathrm{BEC}$, 然后 利用微波场作用于BEC气体原子, 这时会发生从 $|1\rangle$ 状态和 $|2\rangle$ 状态原子之间的拉比转换, 对于一个均匀状态的系统, 每一部分都有相同的波函数相位, 这种转换会以相同的速 度发生在每个部分, 但是由于外加条件, 造成的空间不对 称势的时变性改变了这种互变现象, 这就是用相位印刻方 法产生浴旋的关键。这一不对称势可以由一束远离共振的 激光束围绕着凝聚体旋转来产生, 当微波频率 $\omega_{r f}$ 与激光 束的旋转频率 $\Omega$ 一起作用, 并与 $|1\rangle$ 状态的静止原子到 $|2\rangle$ 状态的旋转原子的过渡频率发生耦合时, 便会使处于 $|2\rangle$ 状的态原子旋转起来, 旋转起来的 $|2\rangle$ 状态的原子处于凝 聚体的边缘, 不旋转的 $|1\rangle$ 状态的原子处于凝聚体的中央, 通过精确控制这种耦合的关闭时间, 便可以得到不旋转的 $|1\rangle$ 状态的原子与旋转的 $|2\rangle$ 状态原子共存的状态, 由于不 旋转的 $|1\rangle$ 状态的原子处于旋转的 $|2\rangle$ 状态原子的中心, 这 样一种结构便会产生涡旋 [12]。这种方法涉及原子成分复 杂, 且浴旋不稳定。

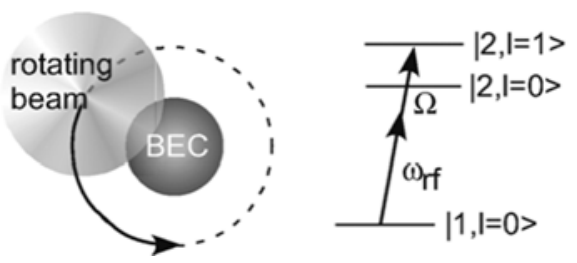

图2 E. A. Corne11小组产生浴旋原理图。

\section{2. 旋转势阱法}

BEC浴旋是超流体的重要特性, 它是超流体应对外界 势阱旋转的一种特殊响应。旋转势阱法可根据势阱的来源 不同划分为旋转激光束法和旋转磁场法。

\section{2. 1 . 旋转激光束法}

\section{(1) 工作原理}

用 “印刻相位法” 在双组分 ${ }^{87} R b$ 的BEC气体原子中产 生浴旋后不久, 法国巴黎Ecole Normale Supérieure院校 Kastler Brossel 实验室的 K. W. Madison、F. Chevy 和 $\mathrm{J}$. Dalibard等人在单一组分的 ${ }^{87} R b$ 中成功实现量子汴旋 [13]。使用的方法与旋转超流体 $\mathrm{He}$ 实验相似, 但本质上又 是有差别的。其工作原理是将原子束缚在一个柱对称的
Ioffe-Pritchard势阱 [14] 中, 一束沿着凝聚体对称轴Z 轴传播的激光在 $Z$ 轴周围以一个比势阱频率高的多的频率 快速振荡, 实际上看起来就像在平衡位置上出现 “两束” 激光, 这 “两束” 激光打破了凝聚体的对称性。因为光束 宽度比凝聚体径向尺寸更大, 所以可以把凝聚体看作是处 在一个轴对称的谐振势和一个非轴对称的谐振势叠加形 成的势阱中, 凝聚体受到的总的势是一个横向的 $(X-Y$ 平 面上）各项异性势（如图3所示）。

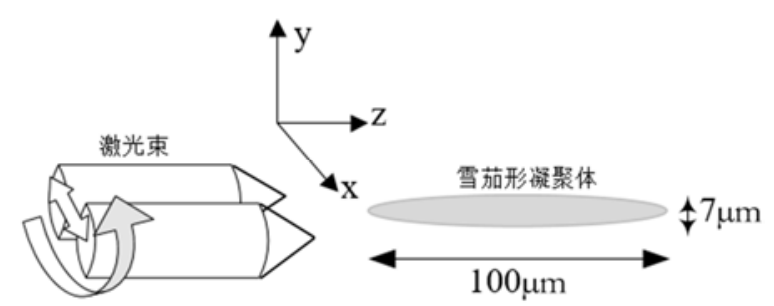

图3 ENS实验原理图。

(2) 实验方法与结果分析

K. W. Madison等人的实验中, 凝聚体温度低于 $80 \mathrm{nK}$, 发生玻色-爱因斯坦凝聚的原子总数为 $N_{0}=(1.4 \pm 0.5) \times 10^{5}$, 图 4为不同 “摚拌” 频率下凝聚体的 截面图, 图中 a, b, c, d对应的激光旋转频率分别为 $145 \mathrm{~Hz}$ 、 $152 \mathrm{~Hz} 、 163 \mathrm{~Hz} 、 168 \mathrm{~Hz}$, a中没有浴旋核出现，b中有一个 浴旋核, $\mathrm{c}$ 中有多个浴旋核, $d$ 中涡旋核正在分解。实验发 现对于给定的原子数和温度, 在某一转动频率以下, 不会 出现浴旋, 通过不断增加旋转频率 $\Omega$, 开始会产生一个浴 旋, 当刚好出现浴旋时的旋转频率叫做临界频率, 实验中 测得的临界频率比理论值高的多, 这是由于原子密度太低 导致 T-F近似（托马斯费米近似）不可靠造成的 [15]。当 旋转频率 $\Omega$ 逐渐增大的时候, 会有更多的涡旋出现, 并形 成浴旋晶格, 但是当旋转频率足够大时, 由于离心势的作 用大于束缚势的作用, $\mathrm{BEC}$ 气体原子将会发生逃逸导致浴 旋消失, 所以浴旋产生的数量与激光 “搅拌” 频率有关。 K. W. Madison等人还给出浴旋数量峰值与对应频率的定量 关系式: $\Omega=0.7 \omega_{\perp}\left(\omega_{\perp}=\sqrt{\omega_{X}{ }^{2}+\omega_{Y}{ }^{2}}\right)\left(\omega_{X}\right.$ 和 $\omega_{Y}$ 分别为 $X$ 和 $Y$ 方向的振荡势, 定义各项异性参数 [15] $\left.\mathcal{E}=\left(\omega_{X}{ }^{2}+\omega_{Y}{ }^{2}\right) /\left(\omega_{X}{ }^{2}+\omega_{Y}{ }^{2}\right)\right)$, 且得出随着各向异性程度 的加深, 浴旋成核数量增大的定性结论。

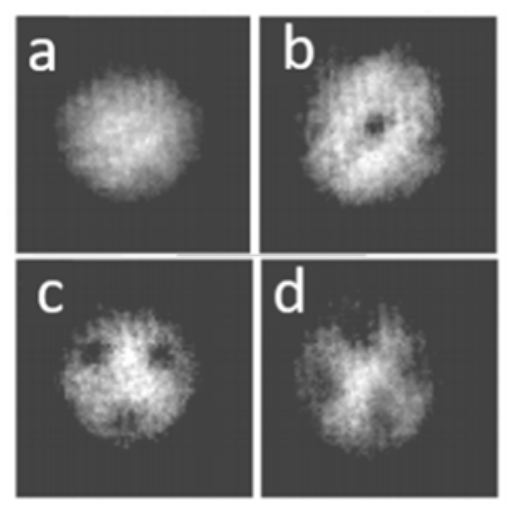

图4 ENS实验结果错误!未找到引用源。。 
近年来, Kastler Brosse1实验室在BEC方面的研究方 向主要有, 超冷状态下的镝原子的复杂状态和新的物理行 为 [16]、外部势阱中磁场参量对于 $\mathrm{Na}$ 原子 $\mathrm{BEC}$ 自旋量的影 响 [17] 以及三维铷原子气体BEC压缩 [18]等。

\subsection{2. 旋转磁场法}

\section{(1) 工作原理}

旋转磁场法 [19] 是将碱金属原子放置于 TOP (Time-averaged orbiting potential) 势阱[17]中, 通 过旋转磁势阱的方式使BEC产生浴旋。

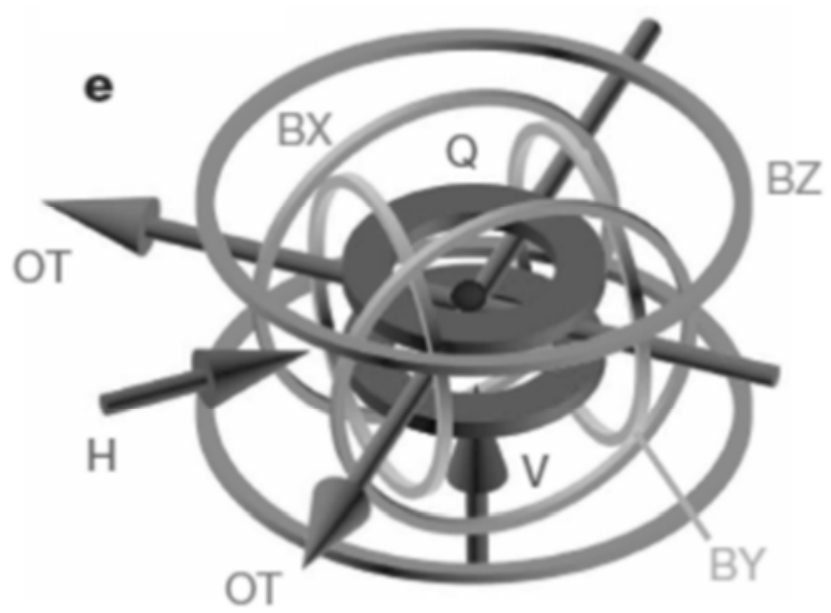

图5 TOP示意图。

TOP势阱由一个球形四极场, 和一个快速旋转的偏置 磁场组成。磁场零区域为径向 (XY) 平面的一个圆环 (如 图5所示), 中间亥姆霍兹线圈产生四极阴, $B_{x}, B_{y}, B_{z}$ 产生X、Y、Z方向的偏置磁场, 用来调整磁场中心位置。 为了使势阱旋转起来, 可以调节偏置场以频率 $\Omega$ 旋转起来, 时变偏置场径向表达式为:

$$
\left(\begin{array}{l}
B_{x} \\
B_{y}
\end{array}\right)=\left(\begin{array}{cc}
\cos \Omega t & \sin \Omega t \\
-\sin \Omega t & \cos \Omega t
\end{array}\right)\left(\begin{array}{c}
E B_{t} \cos \omega_{0} t \\
B_{t} \sin \omega_{0} t
\end{array}\right)
$$

(2) 实验方法与结果分析

对于不同的形变参量, 成核所需的磁阱旋转频率 $\Omega$ 的 范围也不相同 [19]，如表1所示。

表1 $\mathcal{E}$ 参量变化对比。

\begin{tabular}{lll}
\hline$\varepsilon$ 取值 & 最大成核平均数 & 最大成核平均数的频率 \\
\hline 0.084 & 5 & 0.82 \\
0.041 & 5 & 0.75 \\
\hline
\end{tabular}

通过实验观察, 当 $\varepsilon$ 分别取值为 0.084 (黑点) 和 0.041 (白点) 时, 最大成核平均数为 5 和 2 , 最大平均成核的偏 置场归一化旋转频率 ( $\left.\bar{\Omega}=\Omega / \omega_{\perp}\right)$ 为 0.82 和 0.75 , 如图6 所示。这种方式与旋转激光束法相比的优点在于, 能提供 更大范围的各项异性势, 从而降低旋转频率, 但是在操作 复杂, 延时较大。

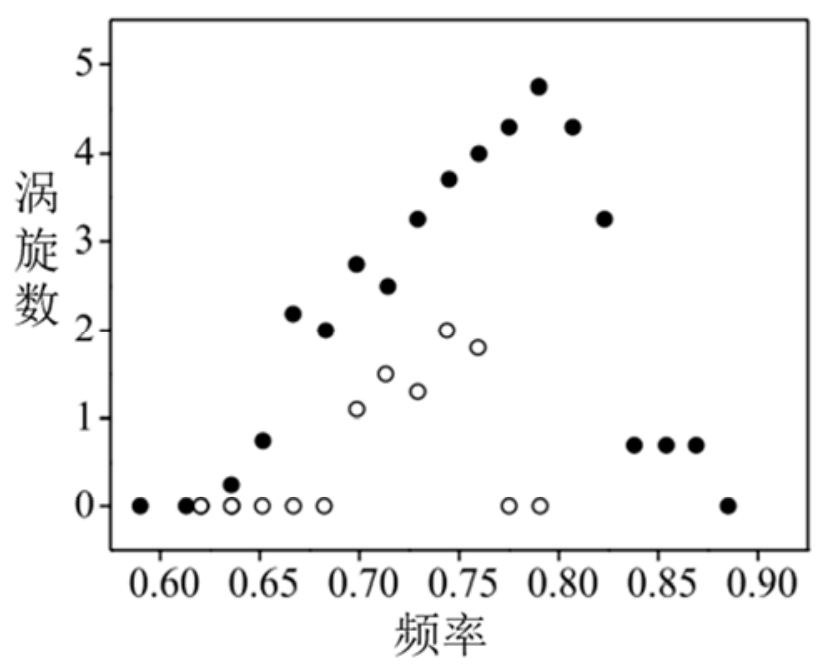

图6 浴旋数量与旋转频率关系图 [16]。

旋转磁场法最早是由牛津大学物理系Clarendon实验 室提出来的。2014年, 该实验室的D Trypogeorgos等人继 续对磁势阱进行深入研究, 设计了一种新型毫米级的超冷 原子磁势阱, 具有较好的磁场梯度操作能力 [20]。

\section{3. 传递角动量法}

\section{（1）工作原理}

传递角动量法的原理就是原子吸收光子的同时, 能够 获得光子的轨道角动量, 使自身产生浴旋。浴旋光是具有 螺旋型相位波前和相位奇点的光束 [21]。浴旋光自身的相 位分布由相位因子决定，在光束传播方向上的截面上, 若 绕浴旋中心一周, 则其光场的相位就改变 $2 \pi l$ ( $l$ 为浴旋 光的拓扑荷数), 并且螺旋形相位的中心为相位奇点 [22], 如图7所示。

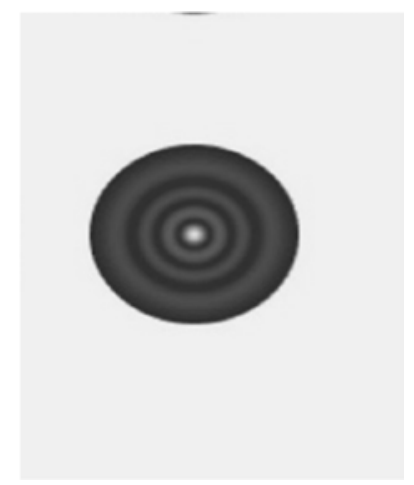

图7 1=1浴旋光光斑图。

光子能够携带两类角动量: 一类是自旋角动量 (SAM, 或称内部角动量); 另一类是轨道角动量 (OAM, 或称外 部角动量）。一束Laguerre-Gaussian ( $L G_{p}^{l}, p$ 为径向 节点数) 光束具有沿着传播方向的轨道角动量, 每个光子 轨道角动量为 $l \hbar$ ( $\hbar$ 为约化普朗克常量)。

(2) 实验方法与结果分析

如图 8 所示 [23]，美国国家标准技术研究所的 M. F. Anderson、C. Ryu等人用相向传播的 $L G_{0}^{1}$ 光束和高斯 
(a) 光束, 作用在BEC原子团上, Probe光为检测光 $(b)$ 。 质量为 $\mathrm{M}$ 的原子吸收一个 $L G_{0}^{1}$ 光子后, 同时放射出一个高 斯光子, 获得 $2 \hbar k$ 的线动量 (其中 $k=2 \pi / \lambda, \lambda$ 为光波长)。 两束高斯光束会发生共振布拉格衍射, 两束光束的频率差:

$$
\delta v=\frac{4 E_{r}}{h}=4 v_{r}
$$

其中 $E_{r}=(\hbar k)^{2} / 2 M$ 是弹性势能, $h$ 为普朗克常量。

通过布拉格衍射转移的线动量可以看做是处于正弦 振荡的光耦极子势的原子通过衍射产生的, 这种光耦极子 势是由反向传播的高斯光束干涉产生的。这里 $L G_{0}^{1}$ 光束和 高斯 $(G)$ 光束干涉产生的光极势是非正弦的, 但是由于 径向紧缩的轮廓和 $L G_{0}^{1}$ 光束的螺旋型相位, 光极势也是螺 旋型的。这种螺旋势的衍射便产生了一种旋转状态。

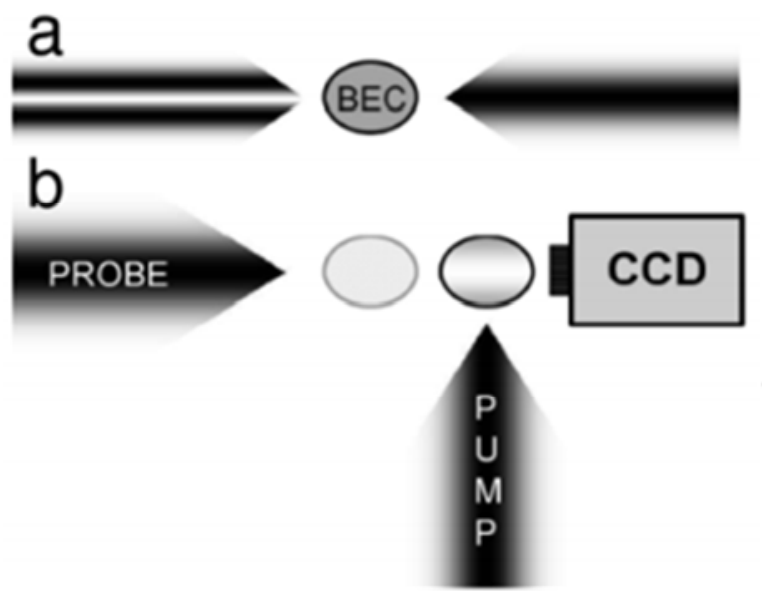

图8 实验装置示意图。

\section{4. 改进型旋转势阱法}

\section{(1) 工作原理}

为克服旋转激光束利用振荡产生各向异性势较弱, 旋 转光束频率要求较高, 而旋转磁场法操作手段较难实现等 不足, 提出了一种提高涡旋产生效率和增强可行性的方法。 在磁阱捕获冷原子系统中, 通过设计四极场中偏置场的各 项参数, 获取势阱较大范围的各向异性, 采用激光旋转势 阱中冷原子形成浴旋, 进而提高量子浴旋的形成效率, 也 简化了实验操作的复杂度。本改进型旋转势阱法与上述旋 转势阱法的主要区别是, 与旋转激光束法相比, 利用磁阱 产生各项异性势, 取代原来利用激光振荡产生各向异性势 的方法; 与旋转磁场法相比, 用激光旋转来操控原子旋转, 克服旋转磁阱的操作实现难题。

(2) 实验方案

势阱频率在 3 个坐标轴上的分量可分别表示为: $\omega_{\mathrm{x}}, \omega_{\mathrm{y}}, \omega_{\mathrm{z}}$, 势阱横向各项异性可表示为:

$$
\varepsilon=\frac{\omega_{\mathrm{y}}^{2}-\omega_{\mathrm{x}}^{2}}{\omega_{\mathrm{x}}^{2}+\omega_{\mathrm{y}}^{2}}
$$

$$
\frac{\omega_{\mathrm{x}}}{\omega_{\mathrm{y}}}-1 \approx \frac{B_{y} / B_{\mathrm{x}}-1}{4}
$$

所以通过设计偏置场幅值 $B_{\mathrm{x}} 、 B_{\mathrm{y}}$ 的值, 即可改变势 阱势的各项异性，从而为下一步激光 “搅拌” 原子做准备。

采用一束蓝失调聚焦激光束, 沿着磁阱对称轴方向传 播, 并且以一定的频率 $\Omega$ 旋转起来。原子在光场的作用产 生感应偶极矩, 并且会受到光场双极子势的作用, 会发生 向光最强处的运动, 由于激光束的旋转, 便会带动原子旋 转并产生浴旋。

\section{4. 总结与展望}

$\mathrm{BEC}$ 量子浴旋的陀螺效应已在理论和实验上得到验证, 具有发展超高灵敏度陀螺仪的巨大优势。本文论述的BEC 浴旋成核方法种类多样, 实际使用中可根据具体应用对象 和工作环境采用相应的BEC浴旋成核方法。随着激光操控 原子技术的发展, 基于浴旋一反浴旋对的叠加干涉效应来 敏感角速率, 理论上也具有超高灵敏度精度的可能性, 值 得一提的是, BEC 浴旋成核方法对浴旋一反浴旋对的叠加干 涉成核研究具有一定的借鉴意义, 为此继续探索BEC浴旋 成核方法具有重大意义。

\section{致谢}

本文受到国家863项目 “玻-爱凝聚态**机理研究” 的 支持。

\section{参考文献}

[1] Garraway B M, Suominen K A. Wave-packet dynamics: new physics and chemistry in femto-time [J]. Reports on Progress in Physics, 1995, 58 (4) : 365-419 (55).

[2] Anderson B P, Haljan P C, Wieman C E, et al. Vortex precession in Bose-Einstein condensates: Observations with filled and empty cores [J]. Physical review letters, 2000, 85 (14): 2857.

[3] 苗元秀. 旋转的原子玻色-爱因斯坦凝聚体的性质 [D]. 清华 大学, 2002。

[4] Davis K B, Mewes M, Andrews M R, et al. Bose-Einstein condensation in a gas of sodium.

[5] Stringari S. Superfluid gyroscope with cold atomic gases [J]. Physical review letters, 2001, 86 (21): 4725 .

[6] Hodby E, Hopkins S A, Hechenblaikner G, et al. Experimental observation of a superfluid gyroscope in a dilute Bose-Einstein condensate [J]. Physical review letters, 2003, 91 (9): 090403.

当 $\varepsilon$ 值在较小范围内时: 
[7] Cozzini M, Stringari S, Bretin V, et al. Scissors mode of a rotating Bose-Einstein condensate $[\mathrm{J}]$. Physical Review A, 2002, 67 (2): 426-430. Davis K B, Mewes M, Andrews M R, et al. Bose-Einstein condensation in a gas of sodium.

[8] Ketterle W. Inside the quantum Hall effect [J]. Nature Physics, 2015, 11 (2):90-91.

[9] Byrnes T, Dowling J P. Quantum Hall effect with small numbers of vortices in Bose-Einstein condensates [J]. Physical Review A, 2015, 92 (2).

[10] Matthews M R, Anderson B P, Hal jan P C, et al. Vortices in a Bose-Einstein condensate [J]. Physical Review Letters, 1999, 83 (13): 2498.

[11] Williams J E, Holland M J. Preparing topological states of a Bose-Einstein condensate $[\mathrm{J}]$. Nature, 1999, 401 (6753) : 568-572.

[12] Aftalion A. Vortices in Bose-Einstein Condensates [M]. Springer Science \& Business Media, 2007.

[13] Madison K W, Chevy F, Wohlleben W, et al. Vortex formation in a stirred Bose-Einstein condensate $[\mathrm{J}]$. Physical Review Letters, 2000, 84 (5) : 806.

[14] Fetter A L. Rotating trapped Bose-Einstein condensates [J]. Laser Physics, 2008, 18 (1):1-11.

[15] Kasamastu K, Tsubota M. Quantised Vortices in Atomic Bose-Einsten Condensates [J]. Progress in Low Temperature Physics, 2009, 16: 351-403.
[16] Dreon D, Sidorenkov L A, Bouazza C, et al. Optical cooling and trapping highly magnetic atoms: The benefits of a spontaneous spin polarization $[\mathrm{J}]$. 2016.

[17] Zibold T, Corre V, Frapolli C, et al. Spin nematic order in antiferromagnetic spinor condensates $[\mathrm{J}]$. Physics, 2015.

[18] Chomaz L, Corman L, Bienaimé T, et al. Emergence of coherence in a uniform quasi-two-dimensional Bose gas [J]. Nature Communications, 2014, 6.

[19] Hodby E, Hechenblaikner G, Hopkins S A, et al. Vortex nucleation in Bose-Einstein condensates in an oblate, purely magnetic potential. [J]. Physical Review Letters, 2002, 88 (1).

[20] Trypogeorgos D, Albright S D, Beesley D, et al. Design of a millimetre-scale magnetic surface trap for cold atoms [J]. Journal of Physics B Atomic Molecular \& Optical Physics, 2013, 47 (7) : 1216-1221.

[21] Petrich W, Anderson M H. Stable, Tightly Confining Magnetic Trap for Evaporative Cooling of Neutral Atoms. [J]. Physical Review Letters, 1995, 74 (17): $3352-3355$.

[22] Drain L E. The laser Doppler techniques [J]. Chichester, Sussex, England and New York, Wiley-Interscience, 1980. 250 p., 1980, 1.

[23] Andersen M F, Ryu C, Cladé P, et al. Quantized rotation of atoms from photons with orbital angular momentum [J]. Physical review letters, 2006, 97 (17) : 170406. 